\title{
Langue et littérature. Les premières anthologies italiennes pour l'enseignement du français XIX $^{\mathrm{e}}$ siècle)
}

Nadia Minerva

\section{OpenEdition}

\section{Journals}

Electronic version

URL: https://journals.openedition.org/dhfles/3066

DOI: $10.4000 /$ dhfles.3066

ISSN: 2221-4038

\section{Publisher}

Société Internationale pour l'Histoire du Français Langue Étrangère ou Seconde

\section{Printed version}

Date of publication: 1 June 1999

Number of pages: p.382-401

ISSN: 0992-7654

\section{Electronic reference}

Nadia Minerva, "Langue et littérature. Les premières anthologies italiennes pour l'enseignement du français (XIXe siècle)", Documents pour l'histoire du français langue étrangère ou seconde [Online], 23 | 1999, Online since 06 July 2015, connection on 10 March 2023. URL: http://journals.openedition.org/ dhfles/3066 ; DOI: https://doi.org/10.4000/dhfles.3066

This text was automatically generated on 10 March 2023.

All rights reserved 


\title{
Langue et littérature. Les premières anthologies italiennes pour l'enseignement du français (XIX ${ }^{\mathrm{e}}$ siècle)
}

\author{
Nadia Minerva
}

\section{Situation de l'enseignement du français en Italie dans la deuxième moitié du XIXe siècle}

Dans la période qui fournit le cadre de ce Colloque, les conditions de l'enseignement du français ont profondément évolué en Italie. Deux facteurs de toute importance sont à signaler : l'institutionnalisation de l'enseignement des langues étrangères en 1859-60 et l'entrée du français dans les cursus des lycées en 1889. Ces facteurs devaient influer de façon décisive sur l'histoire du manuel, sur sa composition et sur sa diffusion.

C'est au moment de l'implantation de l'enseignement des langues modernes dans les écoles publiques que se manifeste l'intérêt des auteurs de manuels pour la littérature française, pour des raisons qui ont été étudiées et que je rappelle brièvement. Sous l'Ancien Régime ou dans la première moitié du XIX ${ }^{e}$ siècle, avant la scolarisation du français, les apprenants maniaient des livres français qui figuraient dès le début de l'apprentissage à côté des livres de grammaire. La tradition des Excerpta, utilisés dans l'enseignement des langues classiques, ne paraît donc pas influencer l'enseignement des langues modernes en Italie et, pour ce qui concerne les manuels d'Ancien Régime, les lectures ou les exercices proposés étaient presque toujours des textes fabriqués. Les auteurs font leur entrée dans lesmanuels quand les établissements d'éducation adoptent l'enseignementcollectif et que l'enseignement du français s'élargit à en, public nouveauprovenant de classes sociales plus basses : ce nouvel élève- dont lapréparation linguistique est déficitaire, même en italien - est juge incapable d'affronter des ouvrages intégraux ; d'où une progression rigoureuse dans la 
présentation des textes authentiques et leur adaptation - sousforme d'anthologie - à la classe de langue.

\section{Le corpus}

Quant aux anthologies que je me propose d'étudier, l'offre de ce génied'outil didactique est remarquable surtout vers la fin de la période étudiée. En effet, si leur nombre est assez exigu avant la disciplinarisation dufrançais pour les raisons qu'on vient de rappeler, à partir des années 60 ce nouveau genre de texte scolaire commence sa progression. Ces manuels, d'abord rares et succincts, deviennent, au fil des années, de plus; en plus nombreux et riches en textes d'auteurs de toutes les époques,parallèlement à l'élargissement du public scolaire : si dans les années 60-90 leursdestinataires, les élèves des écoles et des instituts techniques," n'étaient quefaiblement concernés par la formation littéraire, vers la fin dusiècle, avecl'entrée du français dans les lycées, l'importance de la littérature s'accroîtsensiblement.

Quant aux années 30-60, S. Torretti publie en 1834 des Récréations du cœur et de l'esprit ou recueil de morceaux tirés des meilleurs auteurset», dans la même période, un Manuel de lecture ${ }^{1}$, dont les sujets sontl'histoire sacrée et l'histoire romaine ; dans le Nouveau manuel de lecture dumême auteur, de 1853, les sujets sont tirés de l'histoire grecque, dela mythologie, de la géographie et de l'histoire naturelle; dune, seulle premier des trois textes propose des morceaux d'auteurs.A J.-M, Merle nousdevons des Élémens de lecture française (1835), qui ne font place qu'à La Fontaine, Boileau et Florian. Pour la période qui précède la scolarisation du français on compte une vingtaine d'ouvrages ${ }^{2}$ dont la plupart sont concentrés dans la décennie 50-60 ; ces manuels dépassent rarement les deux cents pages et proposent souvent des morceaux fabriqués ou anonymes. Nous sommes loin de la floraison à laquelle on assiste dans la période suivante.

5 L'analyse des anthologies devrait évidemment se fonder sur tout le corpus des manuels circulant dans la péninsule dans la deuxième moitié du XIX $\mathrm{X}^{\mathrm{e}}$ siècle, mais si le recensement des textes publiés en Italie est fait pour la première partie du siècle, le dépouillement des manuels de la deuxième période est encore en cours : on peut cependant s'en faire une idée sur la base du millier de titres déjà repérés (mais nous sommes encore loin du chiffre final) : si on ne prend en compte que les premières éditions, jusqu'ici nous avons trouvé 184 anthologies et manuels de lecture, publiés avant 1910.

$6 \quad$ Faute d'un corpus exhaustif qui pourrait fournir des données exactes, je me bornerai à l'étude de quelques textes. Parmi les 65 anthologies et livres de lecture que j'ai pu consulter, $\mathrm{j}$ 'en ai choisi quelques-uns qui ont eu une diffusion importante et qui pourraient être considérés comme emblématiques de différentes situations d'enseignement, ou de différentes attitudes vis-à-vis de la littérature. L'hétérogénéité des outils à notre disposition permet de se faire une idée de l'horizon didactique ${ }^{3}$ et de relever les caractéristiques principales de l'enseignement de la littérature et de la culture françaises : le Musée littéraire. Lectures choisies en prose et en vers de Sylvain Gagnière (1866) ne compte que 150 pages, tandis qu'avec La France littéraire de Joseph Poerio (1878) nous avons affaire à un volume de 500 pages $^{4}$; le Nouveau cours de lectures françaises de Podalyre Pétri ni (1882) ne s'adresse qu'aux élèves des Instituts techniques, tandis que le Manuale di lettura de G.D. Prochet (1891) est destiné aussi aux élèves des 
lycées et des écoles normales ; les Exercices de mémoire et de style mis à la portée des enfants de Belèze (1859) est un ouvrage pour la première jeunesse, tandis que les adaptations italiennes des célèbres Leçons de littérature et de morale de Noël et La Place sont évidemment conçues pour les élèves des classes terminales et pour les aspirants professeurs ; la Littérature contemporaine de Ghiotti et Dogliani (1893) ne présente que des auteurs de la deuxième moitié du XIX $\mathrm{X}^{\mathrm{e}}$ siècle,, comme le Français contemporain d'Emile Puget, qui publie aussi des Pages de lecture des meilleurs écrivains français depuis l'origine jusqu'à nos jours (1894);le Libro di lettura francese de Prochet est fourré de questions et denotes phonétiques, morphosyntaxiques et lexicales, tandis que les adaptations italiennes des Leçons de Noël et La Place, aussi bien que les Pages de lectures de Puget (1894), ne proposent que des notes historiques ou littéraires et de rares traductions de mots isolés. La Mosaïque littéraire de Ghiotti et Dogliani (1894) fait place aussi à des morceaux manipules d'auteurs à peine mentionnés ; l'Anthologie française de Malan, augmentée par Serafini (1895) contient, comme plusieurs autres, un précis d'histoire littéraire ; nous avons des volumes austères aux pages remplies de petits caractères serrés et de jolis livrets ornés de gravures comme la Mosaïque littéraire de Ghiotti et Dogliani. Et on pourrait encore continuer à relever les traits en opposition, puisque la typologie des manuels est très variée, surtout en rapport avec les destinataires et avec les dimensions des ouvrages.

7 Ces deux facteurs - étroitement liés et déterminants dans la composition du manuel ne sont pas sans conséquence sur le choix des textes présentés : ils constituent les repères les plus sûrs pour analyser les motivations des auteurs. Par ailleurs, on pourra aussi enregistrer le témoignage d'une évolution de l'idée de littérature et la naissance d'une conscience historique, qui se font jour en dépit des visées surtout linguistiques de l'enseignement du français en Italie.

8 Les pour et les contre des anthologies sont parfois évoqués et ce vieux débat ne cesse d'être réactivé ; au tournant du siècle, Giovanni Bonfiglio se justifie dans la huitième édition de ses Letture francesi scelte ed annotate (1897) : si Bossuet " ne jugeait pas à propos de faire lire à son élève (le Dauphin) les ouvrages des auteurs 'par parcelles' », il peut citer à l'appui l'opinion de Fénelon et surtout l'autorité de Rollin et de Quintilien. Il défend donc la méthode par morceaux choisis, plus simple, plus délassante, qui doit précéder la lecture d'ouvrages complets (Avant-propos).

9 Le terme même d'anthologie n'apparaît pas tout de suite : les premiers textes autonomes se définissent plutôt comme " manuels ", « cours pratiques », « exercices ", « livres de lecture »: ce seront, dans les années 60 , des « recueils » ou des « musées littéraires ", comme ceux de G. Zuliani (Raccolta di racconti, descrizioni, lettere e dialoghi nelle due lingue italiana e francese, 1861), ou de S. Gagnière (Musée littéraire. Lectures choisies en prose et en vers avec notes et notices biographiques, 1866), etc. Par ailleurs, si on propose souvent, avant 1860, des textes fabriqués ou anonymes, dans les années suivantes on préfère des morceaux choisis d'auteurs dont on signale le nom.

\section{L'organisation des anthologies}

Le plan adopté dans les manuels pour la présentation des morceaux, choisis varie suivant quatre options : les morceaux sont classés par genres, par siècles, par utilisation didactique et par matières. 
11 Le classement par genre, on le sait, a un ancêtre illustre : les célèbres Leçonsde littérature et de morale de Noël et La Place, de 1804,, qui ont circulé en Europe pendant toute la première partie du XIX ${ }^{e}$ siècle, sont adaptées aux apprenants italiens en 1862, en 1865 et en $1869^{5}$. Cette modalité die présentation des textes connaîtra une bonne fortune. C'est la formule Noël-De La Place qui est la plus fréquente et c'est elle qui fournit; un modèle prestigieux pour les premières anthologies italiennes. Les textes sont divisés c'est connu- en plusieurs rubriques : tableaux, descriptions, narrations, définitions, allégories, morale religieuse ou philosophie pratique, lettres, discours et morceaux oratoires, caractères ou portraits et parallèles. Le but est de fournir des modèles de style que l'élève italien - comme d'ailleurs le natif - devrait utiliser.

Candido Ghiotti à qui nous devons plusieurs anthologies nous domine aussi un Nouveau Noël et De La Place qui a eu au moins neuf éditions. Podalyre Petrini, dans son Nouveau cours de lectures françaises de 1882 suit fidèlement le classement par genres (Fables et allégories, Histoire naturelle, Petits récits-Narrations historiques, Morale ou philosophie pratique-Définitions, Tableaux-Descriptions, Portraits historiques et parallèles, Lettres classiques, Poésie). La Nouvelle chrestomathie française à l'usage desécoles secondaires et des écoles d'œnologie et de viticulture d'Italiede Ferdinando Bassi, de $1892^{6}$, un ouvrage encore réédité en 1917, adopte ce type d'organisation (Descriptions, Tableaux, Portraits, Parallèles, Narrations, Fables, Paraboles, Discours, Lettres classiques, Poésie etc.), tout en faisant place à un abrégé d'histoire littéraire. Bassi mentionne les titres des ouvrages dont sont tirés les morceaux, fournit de riches notices biographiques sur les auteurs et une table chronologique des principaux écrivains français. D'ailleurs c'est une sorte de compromis entre les deux méthodes, la méthode par genres et la méthode historique, déjà tenté avec l'adaptation des Leçons procurée par Collombey en $1862^{7}$ où un long " résumé de l'histoire de la littérature française » (de 60 pages) précède l'anthologie.

D'autre part, Bassi fait une place importante à des sections plus spécialement consacrées aux exigences de la formation professionnelle : lettres familières et commerciales, modèles d'actes commerciaux et civils, annonces, "Inventions et Découvertes ", "Sciences, Arts, Industries ». Par ailleurs, il divise son ouvrage en quatre parties dans un souci de progression didactique. Bassi est très critique à l'égard de la progression chronologique, pour des raisons didactiques : plusieurs anthologies qui observent l'ordre chronologique, constate-t-il, présentent les morceaux

pêle-mêle sans aucune classification littéraire, de sorte que la plus simple fable de Fénelon ou de La Fontaine se trouve tout auprès d'un morceau philosophique de Descartes ou de Pascal, ou d'une scène tragique de Racine. Cette distribution pourrait convenir aux Français qui doivent connaître le développement littéraire de leur langue, qu'ils étudient onze ou douze ans, comme nous étudions la nôtre, mais non pas aux élèves de nos écoles secondaires, qui n'ont, pour cette étude, que trois ou, tout au plus, quatre heures d'enseignement par semaine, pendant le temps qu'ils fréquentent les écoles et les instituts techniques (p. VI).

Et pourtant, la formule Noël-De La Place est loin de satisfaire tout le monde. En 1859, l'éditeur Gnocchi de Milan propose les Exercices de mémoire et de style mis à la portée des enfants de Belèze, ancien chef d'institution à Paris (ouvrage, précise la page de titre, autorisé par le Conseil de l'Instruction publique en France). Les motivations et les choix de Belèze sont expliqués dans un Avant-propos qui vise à distinguer cet ouvrage des nombreuses anthologies en circulation et qui nous fait aussi comprendre les motivations de l'éditeur milanais : tous calqués sur les Leçons, les recueils de morceaux 
choisis en commerce paraissent destinés à ceux qui connaissent déjà la langue française. Belèze propose au contraire des morceaux qui sont à la portée des jeunes, " soit sous le rapport de la pensée, soitsous le rapport de l'expression » (1859: p. III). Les textes qu'ilpropose sont gradués et les premiers sont plus courts, "plus faciles à comprendre et à retenir ». Le but moral est évidemment tout aussi présent : formerl'esprit et le cœur des enfants par l'exemple de principes moraux inébranlables, par la " beauté des pensées », par la " noblesse des sentiments »; ils'agit cependant de mettre ces principes à la portée des enfants, sous des formes attrayantes, comme les fables et les sujets allégoriques,. Quantà laclassification adoptée, Belèze conteste la classification traditionnelle par genre, "didactique ", « descriptif ", « narratif », etc., souvent arbitraire et inutile pour un destinataire en bas âge, qui n'attache aucun sens à ces divisions : «ce n'est pas d'un ouvrage de rhétorique ou deprincipes delittérature » qu'il a besoin, précise-t-il, mais d'exercices de mémoire sur des modèles de pensée et de style : des poèmes religieux, surtout de Racine, de Jean-Baptiste Rousseau ou de Lamartine ; des fables deLa Fontaine, de Florian, de Berquin ; des vers sur des sujets divers de Boileau, Voltaire» André Chénier, une page des Femmes savantes de Molière, une dePhèdre, une de Cinna ; la prose de Buffon, Fénelon, Bossuet, Massillon, Chateaubriand, etc. Quant aux auteurs, le choix est très varié, même si sur 191morceaux choisis 36 sont de La Fontaine, 30 de Fénelon, 17 de Racine? ${ }^{8}$.

Suivant Poerio (La France littéraire, 1878), lui aussi fort critique vis-à--vis de la formule Noël-De La Place, les anthologies qui partagent les morceaux choisis en autant de groupes qu'il y a de genres littéraires, forment « sans aucune distinction d'époques, des assemblages confus de narrations, de fables, d'allégories, de descriptions, et de tableaux, etc. L'esprit s'égare dans ce pêle-mêle ». D'ailleurs, remarque-t-il, " ces divisions sont souvent arbitraires, car un même morceau peut appartenir à un genre par le but ou le fond, et à un autre par la forme » (Préface). Utiles pour l'enseignement de la rhétorique, ces anthologies ne sont d'aucun intérêt pour l'enseignement d'une langue et d'une littérature étrangères, puisque rien dans ces livres " n'éveille l'attention sur les auteurs dont on lit des fragments; rien ne guide l'intelligence dans les appréciations qu'on serait tenté de faire. L'arrangement des morceaux est lui-même une source d'erreurs et de méprises pour l'étranger. Il lui arrive parfois de prendre Beaumarchais pour un contemporain de Molière, et l'auteur de Gil Blas pour un romancier tout moderne ». Il préfère donc à cette classification " artificielle et stérile ", la méthode historique. Les avantages ne concernent pas seulement la perception correcte du développement littéraire, mais aussi la prise de conscience des caractères propres de la langue d'une période par rapport à une autre, donc des variations qu'elle a subies.

Poerio divise son recueil en deux parties, dont la première présente les écrivains classiques (XVII ${ }^{e}$ et XVIII ${ }^{e}$ siècles) et la deuxième est réservée aux modernes et à l'école romantique. Dans une longue introduction à la première partie, pour laquelle Poerio a utilisé Nisard, Baron, Auguste Brachet, Demogeot, Duruy, Vinet, Roche, Paul Albert et d'autres, il propose un panorama de l'histoire littéraire des origines (avec des notions d'histoire de la langue) jusqu'à Rousseau, tandis que l'introduction à la deuxième partie est tirée de l'Histoire des principaux écrivains français de A. Roche. Des notices sur chaque auteur enfin fournissent des renseignements bio-bibliographiques essentiels.

Les auteurs qui misent sur l'exploitation linguistique des morceaux son nombreux. Certains d'entre eux ne manifestent aucun intérêt pour la dimension littéraire ou 
culturelle de l'apprentissage d'une langue étrangère leur attention ne porte que sur la langue. Dans les Letture graduali francesi de Brian-Rey et Spedini (1868), l'anthologie (Pezzi scelti di letteratura con annotiazioni italiane) constitue la troisième partie du livre. L'indifférence des auteurs à l'égard de l'aspect littéraire du morceau et de la transmission de contenus culturels français est totale : on est en Italie, observent-ils, on s'adresse à déjeunes Italiens, on doit donc présenter les hommes qui ont illustré leur pays (1882 : p. IV) : César, Dante, Galilée, le Tasse, Goldoni. Heureusement, ils font aussi place à de longues pages de Molière (toute une scène de Dom Juan), à des poèmes de Victor Hugo, à la prose de Buffon, de Chateaubriand, etc., pour un total de 64 écrivains dont plusieurs contemporains. Ils donnent aussi - mais pas systématiquement - les dates de naissance et de mort des auteurs cités, ce qui constitue la seule marque d'une attention quelconque au contexte du morceau choisi.

La progression proposée par Bonfiglio (Letture francesi scelle ed annotate per uso delle scuole secondarie d'Italia, 1897) est rigoureusement didactique, modulée, sur les différents degrés de l'école italienne. Une Nomenclatura parlata e scritta sur les centres d'intérêt liés à la vie quotidienne et à de simples notions scientifiques (histoire naturelle, astronomie, météorologie, anatomie...), de petits contes ou des anecdotes, des dialogues, des morceaux choisis faciles de prose et de poésie ; puis la correspondance, privée et commerciale, des pages d'intérêt technique et enfin l'histoire littéraire suivie de contes et nouvelles en version intégrale (d'Edmond About, d'Adrien de Sarrazin, de Guy de Maupassant, d'Eugène Scribe) et des notices sur les auteurs cités dans le manuel. La diffusion de cette anthologie (nous en avons repéré plusieurs éditions dont la dernière est de 1932) fait comprendre que cette disposition des matières, qui rassemble toutes les lectures dans l'ordre des programmes des cursus, depuis les premières classes de l'école technique ou du lycée jusqu'aux classes terminales des instituts techniques, est fort appréciée. Entreprise commerciale, bien sûr, mais aussi une attention toute spéciale' aux exigences de progression dans le choix des textes à proposer.

L'aspect littéraire est négligé aussi dans les anthologies qui suivent une organisation par matières : dans la Nuova crestomazia tecnica francese de Ghiotti et Dogliani (1877), les deux parties proposent respectivement des « Notions utiles de physique, de chimie, de mécanique ", " Machines et outils ", « Principales inventions » et " Le ciel et la terre ", «Les produits des trois règnes de la nature », «Les arts, les métiers, les industries ». Les notices et les précis d'histoire littéraire sont évidemment absents dans ce type d'anthologie, qui proposent cependant plusieurs pages d'auteurs et qui sont aussi destinés aux élèves des lycées.

\section{Notes}

Presque tous les auteurs enrichissent leurs morceaux d'abondantes notes dont la plupart sont des notes de traduction (de mots isolés ou de phrases entières). Dans l'anthologie de Zuliani (Raccolta di racconti, descrizioni, lettere e dialoghi..., 1861) on peut en compter jusqu'à une centaine pour un seul texte. Ghiotti et Dogliani en annoncent 4 000 dans leur Mosaïque littéraire. Ce sont, prétendent-ils, des notes " biographiques, littéraires, historiques "; on a cependant de la peine à dénicher ce genre de notes parmi les milliers de notes linguistiques.

21 Il y a cependant des auteurs qui font exception, chacun pour des raisons différentes. 

vante la clarté et la précision, sont de toute évidence destinées à déjeunes Français, puisque la traduction y est absente : « lis », « serpolet », « lierre », " glaneur » méritent une explication ; "Devant, pour auparavant, ne se dit plus ", écrit l'auteur (p. 14) ; Confucius, Attila, Cerbère, Elisée, Plutarque, Linnée, Jeanne d'Arc, Thésée, Bernardin de Saint-Pierre et plusieurs autres ont aussi besoin de brèves notes. Pour les vers de la fable de La Fontaine, Le coq et la perle : "Un jour, un coq détourna / une perle, qu'il donna / au beau premier lapidaire », l'auteur explique que « Détourner signifie découvrir, trouver » et que "Le poète ne veut pas dire que le lapidaire est beau, mais qu'il se rencontre à propos » (p. 39). Pour les Femmes savantes il précise que «Molière [...] ne veut pas dire que les femmes ne doivent pas être instruites ; il blâme seulement les femmes qui, pour le plaisir d'étaler leur science, négligent les affaires de leur maison » (p. 119).

La Nouvelle chrestomathie française à l'usage des écoles secondaires et des écoles d'œnologie et de viticulture d'Italie de Ferdinando Bassi abonde en notes historiques, géographiques, littéraires, morphologiques, syntaxiques et lexicales, en français. Une table alphabétique des principales notes occupe les huit dernières pages du volume; on signale, avec un système d'abréviations, le domaine d'intérêt de la note qui est tantôt littéraire, tantôt linguistique : acceptions différentes d'un mot dans les deux langues, citations, définitions, grammaire, homonymes, synonymes, paronymes, gallicismes, phraséologie et proverbes. Les définitions par exemple peuvent se référer aux genres présentés :

La. description est lareprésentation par le langage d'un objet physique, d'un, site, d'une scène de lanature, en un mot d'en, phénomène quelconque. Elle prend le nom de description d'après nature, si elle reproduit l'image d'un objet réel ; de description de fantaisie ou d'imagination si elle est la représentation,, d'un objet idéal ; de description scientifique ou technique, lorsqu'elle a. pour but unique d'instruire, de faire connaître les objets dont elle parle, non seulement dans; leur forme extérieure, mais dans, leurs caractères essentiels et constitutifs (1892: p. 37).

Les définitions peuvent aussi concerner des faits de langue : « On appelle gallicismes (gallicismi, francesismi) certaines locutions propres et particulières à la langue française, contraires bien, souvent, aux règles de la grammaire, mais autorisées par l'usage. On; ne pourrait: traduire littéralement ces idiotismes dans une autre langue, sans commettre des gallicismes " (1892; p. 46) ${ }^{9}$. Quant aux notes linguistiques, ce sont les verbes irréguliers qui. sont; souvent ciblés: pour " Pas un seul ne se tait ", par exemple, il fournit la traduction, l'infinitif du verbe (" se taire, v. irr., tacersi, tacere ») et le proverbe : «Qui se tait consent».

Finalement, si les notes historiques et littéraires ne manquent pas, ce sont les notes linguistiques qui se taillent la part du lion. Celles-ci ont parfois l'ambition de se substituer au dictionnaire et fournissent, après la définition, des exemples d'emploi. " Toucher à » (« toccare » est en italien un verbe seulement transitif) est expliqué par P. Petrini de la façon suivante : « porter la main sur quelque chose. Ne touchez pas à cela. Phr. Faire toucher une chose au doigt et à l'œil : la démontrer clairement. Toucher le piano, jouer du piano. Toucher au port, à sa dernière heure » (1882 : p. 99). Il ne néglige cependant pas l'information scientifique quand le texte risque d'être interprété littéralement par les faibles esprits des élèves et qu'il peut donc leur transmettre des notions fausses : par exemple, là où Fénelon, en décrivant un couchant, écrit que le soleil tourne autour de la Terre, Petrini explique que «Fénelon a voulu parler du 
spectacle apparent » (1882:18). Les notes culturelles, moins abondantes, fournissent de courtes explications sur la Bastille, l'invention de l'imprimerie, la Renaissance, les personnages historiques ou les lieux mentionnés dans les morceaux, quelques écrivains...

C'est encore la langue qui est au cœur des notes de Prochet : à son avis le but d'un bon livre de lecture est d'apprendre à lire et de faire appliquer les règles de la grammaire ; ses notes consistent surtout en questions linguistiques de difficulté croissante ; phonétique et orthographe dominent dans les notes des premiers morceaux, morphologie et lexique dans les dernières pages. Pourquoi cet accent ? demande-t-il pour à préposition. Quels sont les rares mots français qui ont un accent sur le a ? (p. 1). La forme haïssait lui fournit l'occasion de demander le subjonctif présent, le participe présent et l'impératif du verbe haïr et le syntagme " au fond de la main » lui permettra de poser une question sur la différence entre fond et fonds au singulier. Le célèbre «Au voleur ! Au voleur ! » de l'Avare de Molière ne servira qu'à faire former le féminin de " voleur, accusateur, empereur et gouverneur » (p. 25), tandis que « Le Coche et la Mouche » de La Fontaine lui suggère des questions comme : quel est le diminutif de mouche ? Combien de mots ont deux v en français? Quand doit-on mettre un s à cent ? (p. 90). Telle page des Martyrs de Chateaubriand ou tel passage extrait de L'Universalité de la langue française de Rivarol n'auront que lemême but : larévision de la grammaire, aucune note littéraire ne distrayant l'élève de cettetâche.

\section{Contenus des morceaux choisis}

Dans nos manuels il s'agit avant tout de fournir des modèles moraux et culturels, et des modèles linguistiques.

Généralement on propose des fables, des textes avec un contenu moral, religieux, historique ou scientifique, des poèmes, des scènes de théâtre... : la morale, l'histoire, les sciences naturelles, les récits de voyages, les vies exemplaires, la littérature pour la jeunesse sont au service de la formation générale du « cœur » et de l'« esprit » des jeunes auxquels on fournit des modèles éthiques, idéologiques et linguistiques, ou bien au service d'une culture de spécialité, vu que, du moins tout au début, jusqu'à la dernière décennie du siècle, cet enseignement ne concernait que les élèves des écoles et des instituts techniques.

L'enseignement " moral » que les Leçons de Noël et La Place garantissent est fort apprécié, d'où leur fortune comme modèle des anthologies italiennes dont les auteurs professent un attachement rigoureux à la vertu et à la morale et qui affichent de tenir surtout en compte l'âge des apprenants.

Un problème de choix assez délicat se présente àceux qui proposent la littérature de leursiècle, commeGhiotti et Dogliani (Littérature contemporaine, 1893) ou EmilePuget (Français contemporain. Morceaux choisis d'auteurs enlevés à la littérature de 1848 à 1869, de 1870 à 1895 et d'auteurs vivants, 1895). Ghiotti et Dogliani déplorent l'absence des contemporains dans les anthologies courantes, donc l'absence de spécimens delangue actuelle, vivante. La hardiesse des idées de ces écrivains, la peinture de passions engendrées par une morale déchue, les formes littéraires qui ressentent de lanévrose dominante ont éloigné ces modernes des pages des manuels. Mais " le tact del'enseignant éducateur " sauraséparer la partie saine, irréprochable decette littérature des pages «non présentables ». La condamnation morale de quelques 
écrivains a fortement conditionné les choix des auteurs d'anthologies. La censure s'exerce par exclusion d'un auteur, par occultation de certaines parties de son ouvrage, par suppression de certaines phrases, puisqu'il s'agit d'offrir ces textes à la lecture des élèves, en classe.

C'est encore la langue de la seconde moitié du siècle qui intéresse Puget, « le français contemporain, si riche, si varié » et il avoue que son choix " a été fait sans aucune préoccupation littéraire, à tel point que [quelques morceaux] ne représentent pas la vraie manière, le caractère dominant des écrivains ". D'ailleurs les programmes recommandent d'enseigner la langue courante. Il s'agit donc de fournir un modèle utilisable, d'où l'intérêt pour les écrivains les plus récents. Le morceau choisi est l'autorité et la source d'exercices de langue.

L'anthologie de Zuliani (Raccolta di racconti, descrizioni, lettere e dialoghi, 1861) propose, parmi les pages des "meilleurs écrivains français ", trente morceaux du Télémaque et trente des Fiancés d'Alessandro Manzoni pour l'exercice de la traduction (version et thème), dans le but de permettre à l'élève de se familiariser avec le style des classiques, de s'approprier l'« esprit » de la langue française et d'en mesurer la « distance » de sa propre langue maternelle (p. 116) ${ }^{10}$. D'ailleurs ce texte est conçu par l'auteur comme une « continuation » de sa propre grammaire (qui n'a abordé, précise Zuliani, que les aspects «mécaniques » de la langue); comme celle--ci, il est partagé en leçons où la progression didactique est rigoureusement respectée et les morceaux français (authentiques ou fabriqués) destinés à la version sont présentés comme " exercices pratiques »; enfin, toutes les notes sont de traduction, ce qui renforce l'aspect de " manuel de langue » de ce recueil.

Ce souci linguistique influence aussi le choix du genre littéraire. Le genre préféré par les auteurs de manuels est la prose. Saint-Ange de Virgile et Mazzucchelli, les éditeurs italiens du manuel de Noël et La Place, nous expliquent que la prose est plus utile au but essentiellement linguistique de l'apprentissage, le morceau choisi servant à l'exercice de la lecture et de la traduction (p. 4); d'où leur décision d'éliminer bien des pages poétiques dans leur adaptation des Leçons. Mais il ne s'agit pas seulement de lire et de traduire. Brian-Rey et Spedini (1882 : p. IV) recommandent un programme didactique qui prévoit trois exercices : répétition, traduction, transcription avec changement de genre, nombre, personne de quelques formes marquées d'un astérisque. Parmi ces derniers figurent des pages de Chateaubriand, des fables de La Fontaine, des poèmes de Victor Hugo, etc.

\section{L'éveil de la conscience littéraire}

Si tout au début de la période analysée nous ne trouvons que des textes édifiants ou didactiques (choisis non pas pour leur valeur littéraire, mais pour initier les élèves au style français tout en formant leur sentiment moral), l'approche historisante finira par prévaloir. Au fil des années, se manifestent les signaux d'une plus grande attention portée au phénomène littéraire d'une part, et à l'altérité de la culture enseignée d'autre part : les auteurs sortent de l'anonymat et la « francité » du texte proposé est de plus en plus mise en vedette.

On indique donc souvent le nom de l'auteur du morceau choisi, on signale ses dates de naissance et de mort. Parfois nous trouvons des index sous forme de dictionnaire biographique des auteurs. En 1880 J.-J. Garnier publie des Modèles d'analyse et de critiques 
littéraires où il explique aux professeurs et aux élèves italiens ce qu'il faut entendre par analyse littéraire -un exercice nouveau pour l'école italienne dans le domaine des langues modernes - quelles sont ses techniques et ses finalités. Il faut, explique-t-il, que la littérature française soit, étudiée avec la même profondeur avec laquelle on s'applique en Italie à la littérature ancienne, parce que le résultatde l'étuded'une langue doit être la connaissance non seulement de "son génie grammatical », mais encore de "son génie littéraire » (Introduction, p. XI).

Dans les Pages détachées de littérature française de Tito Zanardelli, l'attention au fait littéraire prime sur les préoccupations d'éducation morale :

Une anthologie étant surtout une oeuvre impersonnelle» écrit-il, c'est avec unesprit d'impartialité au-dessus de tout soupçon qu'on -a ici accueilli des morceaux d'auteurs professant tour à tour toutes les opinions politiques etreligieuses, pourvu qu'elles fussent soutenues ou justifiées par des talents incontestables.

Aucune surprise donc s'il héberge le Voltaire conteur, le Rousseau du Contrat social, ou des histoires scabreuses ou-abracadabrantes comme Manon Lescaut, La Religieuse ou Le Diable amoureux (le passageoù Biondettase transforme en diable sous les yeux d'Alvare). Dans cette optique de valorisation de la littérarité, les données bio-bibliographiques ne suffisent plus ; «Les notices biographiques ne visant qu'à l'homme, et les énumérations des œuvres d'un auteur n'en faisant connaître que le côté le plus extérieur, les unes et les autres ont été remplacées pardes résumés critiques ", écrit-il dans sa Préface (p. 4).

Dans Atravers lalittérature française contemporaine d'E. Fioreratino (mais nous sommes déjà en 1909), l'auteur regrette de ne pas pouvoir offrir aux élèves de l'école italienne toute l'œuvre des principaux auteurs (ceserait, pense-t-il, la meilleure manière d'aborder la littérature), mais il nous assureque le choix n'a été nullement arbitraire, puisque les morceaux «caractérisent nettement la manière des auteurs " proposés (p. V). Certaines " Notices littéraires " sont particulièrement soignées; elles établissent des liens entre les auteurs et n'hésitent pas à souligner les traits les plus anticonformistes des écrivains présentés. Une place importante est faite à des poètes comme Baudelaire ou Verlaine, ou aux débats sur la littérature, d'où la présence d'auteurs comme Mme de Staël ou Sainte-Beuve ; le titre de l'ouvrage d'où le morceau est tiré est toujours signalé.

Ce n'est donc que sur la durée d'un demi-siècle qu'on assiste, dans les manuels, d'une part à l'éveil de la conscience littéraire, d'autre part à l'implantation de la notion d'histoire littéraire. Mais cet éveil se noue, dans l'école italienne, avec une valorisation de la culture littéraire au détriment des compétences linguistiques qui finissent par intéresser de moins en moins.. Le procès d'ennoblissement des langues modernes, on le sait, passe à travers les belles-lettres. L'enseignement littéraire finira par prévaloir, marginalisant l'apprentissage linguistique, d'abord dans les écoles de la formation classique, et ensuite, paradoxalement, aussi dans les cursus de la formation technique. C'est à ce. début d'une nouvelle époque que nous, font assister nos, anthologies fin de siècle. 


\section{BIBLIOGRAPHY}

AMANTE, Bruto (1.901), Nuovo codice scolastico vigente. Leggi, decreti regolamenti, circolari e programmi dal 1859 al 1901, Roma.-

BASSI, Ferdinando (1892), Nouvelle chrestomathie française à l'usage des écoles secondaires et des écoles d'œnologie et de viticulture d'Italie, Trévise,. Zoppelli.

BELÈZE, G. (1.859), Exercices de mémoire et de style mis à la portée des enfants, Milan, Jacques Gnocchi. BONFÏGLIO, Giovanni (1.897), Letture francesi scelte ed annotate per uso delle scuole secondarie dltalia, Caltanissetta, presse l'Autore.

BRIAN-REY et SPEDINI (1868, éd. consultée 1882), Letture graduali francesi Lettere famigliari e di commercio. Pezzi scelti di letteratura con annotazioni italiane e regole dipronuneia, Firenze, pressoBrian-Rey e Spedini.

COLlOMBEY, P.A. (1862), Leçons françaises de littérature et de morale sur le plan de MM Noël et De La Place, Turin, Libr. De la Minerve: Subalpine.

FIORENTINO, E. (1909), A travers la littérature contemporaine. Notices littéraires et morceaux choisis des auteurs français du $\mathrm{XX}^{e}$ siècle, Florence, Sansoni.

GAGNIÈRE, Sylvain (1866), Musée littéraire. Lectures choisies en prose et en vers avec notes et notices biographiques, Florence-Turin-Milan, Paravia.

GARNIER, Jean-Joseph (1880), Modèles d'analyse et de critique littéraire, Torino, Paravia.

GHIOTTI, Candido (1880), Nouveau Noël et De La Place ; leçons françaises de littérature et de morale, Torino, Paravia.

GHIOTTI, Candido / DOGLIANI, Giustino (1893), Littérature contemporaine. Lectures françaises tirées la plupart des meilleurs écrivains de la dernière moitié du XIX ${ }^{e}$ siècle, graduées et accompagnées de nombreuses notes explicatives, biographiques, historiques à l'usage des écoles secondaires d'Italie, Turin, Paravia.

GHIOTTI, Candido / Dogliani, Giustino (1894), Mosaïque littéraire. Livre de lecture à l'usage des écoles secondaires d'Italie, Torino, Petrini.

MALAN, Jean-Pierre (1895), Anthologie française à l'usage de l'enseignement secondaire. Nouvelle édition augmentée et enrichie par Silvio Serafini, Città di Castello, Lapi.

MINERVA, Nadia / PELLANDRA, Carla (dir.), Insegnare il francese in Italia. Repertorio analitico di manuali pubblicati dal 1625 al 1860, Bologna, Patron, 1997.

PETRINI, Podalyre (1882), Nouveau cours de lectures françaises choisies et graduées à l'usage des instituts techniques (troisième partie, cours supérieur), Milan, Galli et RaimondL

POERIO, Giuseppe (1878), La France littéraire ou les prosateurs et les poètes français depuis Pascal et Malherbe jusqu'à nos jours, Napoli, Pellerano.

PROCHET, G.D. (1891, éd. consultée : 1907), Manuale di lettura francese corredato da numerosissime domande in applicazione delle regole grammaticali, Torino, Paravia.

PUGET, Emile (1894), Pages de lecture des meilleurs écrivains français depuis l'origine jusqu'à nos jours, Roma, Cecchini. 
PUGET, Emile (1895), Français contemporain. Morceaux choisis enlevés à la littérature de 1848 à 1869, de 1870 à 1895 et d'auteurs vivants à l'usage des écoles italiennes, Roma, Cecchini.

SAINT-ANGE de Virgile / MAZZUCCHELLI, G.B. (1869), Leçons de littérature et de morale par MM. Noël et De La Place. Nouveau choix de prose et de vers adapté à l'usage des maisons d'éducation d'Italie, et des jeunes gens des deux sexes qui étudient la langue française, Milan, Sonzogno.

ZULIANI, Giuseppe (1861), Raccolta di racconti, descrizioni, lettere e dialoghi nelle due lingue italiana e francese colla guida delle parole più difficili per la vicendevole traduzione, Verona-Milano, Civelli.

ZANARDELLI, Tito (s.d.), Pages détachées de littérature française, précédées de résumés critiques et accompagnées de notes, Milan-Palerme-Naples, Sandron.

\section{NOTES}

1. Ce texte a connu plusieurs rééditions, dont la dernière repérée est de 1859 ,

2. Je cite encore quelques titres : P.-A. Brochet, Premières lectures françaises, 1838 ; De Clugny, Traitaio elemeniare di pronunzia francese preceduto da una scella di massime e di squarci di prosa ricavati da più celebri autori francesi, 1840 ; Anonyme, Cours de lectures françaises 1841 ; Anonyme, Modèles de style à l'usage de la jeunesse, 1841 ; E. Le Monnier, Cours de langue française pratique, 1849 ; Villemard, Cours pratique de langue et de littérature françaises, 1850 ; A. Boniface, Mosaïque littéraire, 1851 ; J. Madia, Morceaux choisis de lecture française, 1851 ; J. Campana, Lectures françaises, 1852 ; F. Ferrante, Pezzi scelti di autori francesi, 1853 ; Ch. Musslin, Premiers exercices de lecture, 1853 ; G. Asti, Petit musée, 1854 ; G. Moneta, Prima lettura in lingua francese, 1854 ; G. Belèze, Exercices de mémoire et de style, 1859 ; J.-A. Fornier, Récréation, 1859 ; Ch.-H. Rossari, Second livre de lectures françaises, 1860 ; L. Schor, Premières lectures françaises, 1860. Pour les références complètes et l'analyse de ces anthologies, voir Minerva /Pellandra dir. (1997).

3. Sur l'insuffisance du témoignage fourni par l'analyse des anthologies ou par d'autres manuels pour évaluer la complexité de l'enseignement littéraire en classe, je me permets de renvoyer à mon étude ; Problèmes de méthode. Pour un recensement des auteurs français dans les manuels, à paraître dans Documents, $\mathrm{n}^{\circ} 22$,

4. La page de titre de l'édition consultée (1878), la quatrième, signale que l'ouvrage a obtenu une médaille au Congrès pédagogique de 1871 , ce qui nous renseigne sur la date approximative de la première édition non repérée.

5. Celle de 1869 est la sixième édition procurée par 1 imprimeur Sonzogno de Milan. Nous n'avons pas repéré les précédentes ; une édition de $1 \& 4 \mathrm{I}$ est mentionnée dans une liste de titres ayant trait à l'enseignement $\mathrm{du}$ français, qui figure dans une édition du Manuel de lecture de Torretti de 1846.

6. J'ai consulté la « deuxième édition revue et augmentée ». On en connaît une de 1891.

7. Une note écrite à la main sur la page de titre de l'exemplaire consulté nous informe que la première édition remonte à 1847 .

8. Dans la section «Poésie » sont présents des auteurs comme Corneille,, Malherbe;Louis Racine, Le Franc de Pompignan, Mme Tastu, Florian, Reboul, Giniaud ; ily figure des fables (de La Fontaine, Florian, Amault, Berquin», Aubert, LeBailly; Dorat)des sujets divers de Mme Tastu, Arnault, Delille, De Jussieu, Voltaire, Thomas Racan, Gilbert, Soumet, Andrieux, Collin d'Harleville, Guiraud, Ducis, Sedaine; Casimir Delavigne, La Fontaine, etc. et aussi un extrait d'Athalie. La prose est présente avecLamartine, Blanchet, Saint-Lambert, Chamfort, Maury, Mmede Maintenon, Barthélémy, Villemain, Florian, le Cardinal de Bausset, Aimé Martin, une page de la Bibliothèque orientale, Guizot, Michaud, Thomas, Mme de Sévigné, Bernardin deSaintPierre, Filon, Lacépède. 
9. «Par exemple, le gallicisme : Comment vous portez-vous ? il faut traduire en italien : Come state ? et non pas : come vi portate ?, car dans ce dernier cas, on commettrait un gallicisme (francesismo) ».

10. «Siccome il miglior mezzo per impadronirsi dello spirito di una lingua è quello di rendersi famigliare lo stile dei classici autori della stessa, e di conoscere la distanza che esiste tra quelli ed i classici della propria nazione, abbiamo scelto alcuni brani del Fénélon (sic) per la traduzione italiana ed altri del Manzoni per la traduzione francese » (p. 116).

\section{AUTHOR}

\section{NADIA MINERVA}

Université de Bologne 